\section{Hats and gloves off}

The following observations are written more as a 'private citizen' than as editor because they reflect a personal bias. This bias arises from my longstanding concerns about parent counselling or education to prevent chilhood injuries. These concerns were resurrected when I reviewed the paper by Roberts in this issue (p263). Roberts is in good company: the views he expresses about the value of counselling are widely shared and I may well be in a minority in questioning his assumptions regarding its value in this context.

I was sensitized to this issue because I had been asked to write an editorial for another journal on a topic that troubles many of our readers - the occurrence of clothing drawstring strangulations in countries where such clothing has not been banned. ${ }^{1}$ Two such cases occurred in Canada recently, and a paper on the subject was written by respected colleagues. ${ }^{2}$ What struck me about that paper, as well as that of Roberts', is their call for more involvement on the part of health professionals to counsel about this danger and others like it. In the case of the Roberts' paper, the counselling relates to the importance of smoke detector installation and maintenance, but both papers have a common thread: the choice between this strategy and a tougher, regulatory approach.

As I wrote in the Canadian Medical Association fournal editorial, I have three objections to parent education as a stand alone strategy. First, I remain unconvinced that physician counselling or educating is as effective as some assert. Recall the debate in the Opinion/Dissent part of an earlier issue between Bass and Klassen (Sept 1995). Clearly, I agree more with Klassen who expressed doubts about the strength of the evidence. But, even if well motivated, well trained physicians or nurses were as effective as Bass believes, they can only reach a fraction of their patients.

The late Archie Cochrane, a famous British epidemiologist, pointed out that even an intervention that works under ideal circumstances (efficacy), may not work equally well in real life (effectiveness). For example, a new medicine may be efficacious among highly compliant patients, but in the real world many patients are far from fully compliant. Counselling by health professionals is analagous: even if it worked extremely well, it can only work with those who come into contact with a physician or nurse. In injury prevention there is also an added element of urgency. Once it is clear that something is dangerous, parents should be informed promptly. But in a practice situation this can take weeks, months, or even years because only infants and very young children are seen routinely when they are well. Thus, all who do not see a physician for some time may not receive the important message that, for example, drawstrings are dangerous or that smoke detectors save lives.

Few physicians are able to send notices to all families in their practice because, in many instances, without going through records by hand, many may not even know who all their patients are! Granted, some practices such as those that have attached health visitors are more easily able to deliver messages to families who do not visit the physician, but even they are likely to find it difficult to reach all 'at risk' families in a reasonably short period of time.

My third concern about placing undue reliance on physician counselling or education is that it places the main burden on the parent and thus implicitly removes it from the manufacturer or retailer. It is, in effect, an easy way for governments, sensitive to the reactions of industry, to get themselves off the hook. Rather than taking the courageous and possibly politically unpopular step that Britian did in 1969, and ban all clothing with drawstrings, many countries like Canada and the US still rely on the voluntary cooperation of manufacturers and retailers or, even more foolishly, on flawed assumptions about parent education.

Counselling is flawed not only for the reasons I have given, but also because the first step in the process requires that safety notices be received and read by health professionals. Yet, to the best of my knowledge, no one has ever determined the extent to which these messages are read, let alone acted upon in the manner in which they must be if they are to be truly effective.

Finally, the counselling approach worries me because responsibility ends up being placed almost exclusively on the shoulders of families. It is another form of 'victim blaming' that many, including Roberts, rightfully deplore.

It may seem strange that I juxtaposed the smoke detector issue and the drawstring problem. But I do so because many jurisdictions have smoke detector legislation and there is no reason why, as a public health measure, this should not apply everywhere in the UK. Conversely, as stated, the UK has long since banned clothing with drawstrings and this action should be repeated immediately everywhere.

As an international journal, we have a heavy obligation to learn from the experiences of others. This is not the Olympics; there is no room for national pride such that each country must discover for itself, not only the problem, but also the solutions to it.

1 Pless IB. Childhood injury prevention: time for tougher measures. Can Med Assoc F 1996; 155: 1429-31.

2 Petruk J, Shields E, Cummings GE, Francescutti LH. Fatal asphyxiations in children involving drawstrings on clothing. Can Med Assoc F 1996; 155: 1417-9.
News that the World Health Organization (WHO) intends to destroy its remaining stocks of smallpox virus is a landmark. Less than 30 years ago smallpox was endemic in 30 countries and affected nearly 15 million people each year, killing about two million. Then WHO set out to vanquish this disease and, through a concerted international effort, it succeeded. In 1980 smallpox was declared the first diease to be 'totally eradicated'. If this can be done for a disease of this magnitude, think what we could do about injuries with the same sort of effort? 